\title{
Desigualdad y exclusión educativa en contexto de pandemia: Desafíos desde México
}

DESIGUALDADE E EXCLUS ÃO EDUCATIVA EM CONTEXTO DE

PANDEMLA: DES AFIOS DESDE O MÉXICO

ALEJANDRo LÓPEZ DE LARA MARÍN

Resumen

El presente artículo realiza un análisis sobre el impacto que tiene la pandemia en la educación en México, partiendo de las consecuencias que el neoliberalismo dejó por más de tres décadas en el Sistema Educativo Nacional, sobre todo en la pérdida del sentido de lo público, así como también plantear cuáles son las alternativas que el Estado mexicano estaría planteando para enfrentar la crisis y sobre todo para avanzar en una transformación fuera del enmarque neoliberal y finalmente desentrañar los elementos de libre mercado que persisten en las instituciones educativas y que buscan tomar fuerza en el contexto de pandemia, las cuales agudizan la exclusión y desigualdad.

Palabras clave:

Educación, Pandemia, Exclusión y Neoliberalismo

Resumo

O artigo apresenta uma análise do impacto da pandemia na educação no México, a partir das consequências que o neoliberalismo deixou por mais de três décadas no Sistema Nacional de Educação, especialmente na perda do sentido do público, bem como leva em conta as alternativas que o Estado mexicano estaria considerando para lidar com a crise e, sobretudo, para avançar em uma transformação fora do marco neoliberal e, finalmente, desvendar os elementos do livre mercado que persistem nas instituições de ensino e que buscam ter força no contexto da pandemia, que exacerbam a exclusão.

Palavras-chave:

Educação, Pandemia, Exclusão e Neoliberalismo

Abstrac

This article conducts an analysis of the impact of the pandemic on education in Mexico, based on the consequences that neoliberalism left for more than three decades in the National Education System, especially in the loss of the sense of the public, as well as to

${ }^{a}$ Doctor en Estudios Latinoamericanos por la Universidad Nacional Autónoma de México (UNAM)

Rev. Caminhos da Educação: diálogos, culturas e diversidades, Teresina, v. 2, n. 2, p.5-21, mai./ago. 2020 
consider what alternatives the Mexican State would be considering to deal with the crisis and above all to advance a transformation outside the neoliberal setting and finally unraveling the free-market elements that persist in educational institutions and seek to gain strength in the context of pandemics, which exacerbate exclusion and inequality.

Keywords:

Education, Pandemic, Exclusión y Neoliberalism

\section{Introdução}

En el siglo XVI, la población del México antiguo se enfrentó a una de las más terribles dificultades que su mundo había conocido: La invasión, en su mayoría de ambiciosos mercenarios, que se creyeron dueños de aquello que desconocían. La llegada de los europeos produjo el exterminio de la población y la destrucción de cosmogonías y saberes, eso que denominó Boaventura de Sousa como epistemicidios. (De Sousa, 2010)

El sistemático sometimiento colonial, se extendió rápidamente y con él, la llegada de enfermedades que contribuyeron a que la población fuera diezmada. Las epidemias y pestes que llegaron al territorio formaron parte de los intentos por destruir el sistema comunal que construyeron por siglos las culturas prehispánicas, no sólo por la enfermedad, sino por las consecuencias sociales.

Los códices ${ }^{1}$, nos hablan de los efectos desgarradores que provocaron la desarticulación de pueblos enteros azotados por las epidemias; percibidas como parte de la invasión y no como hechos aislados, debido a la ventaja que sacaron los europeos sobre ellas. Las hambrunas provocadas por la expansión de cocoliztlí2, fueron la punta del iceberg de una extensa lista de males que minaron la organización social y cultural de los pueblos originarios. Por ejemplo, uno de los espacios más vulnerados fue aquel donde se creaba, compartía y socializaba el conocimiento. La sabiduría de los pueblos originarios no solo fue negada, sino que se convirtió en el blanco de la destrucción. La racionalidad europea intentó desmantelar eso que los mexicas consideraban como Ixtlamachiliztli: la acción de dar sabiduría a los rostros ajenos. (León Portilla, 1957)

${ }^{1}$ Por ejemplo, el Códice Mendocino, el cual puede consultarse en Instituto Nacional de Antropología e Historia https://www.codicemendoza.inah.gob.mx/index.php?lang=spanish (Consultada el 20 de junio de 2020)

2 En náhuatl significa epidemia y era la manera de nombrar a la peste.

Rev. Caminhos da Educação: diálogos, culturas e diversidades, Teresina, v. 2, n. 2, p.5-21, mai./ago. 2020 


\section{Desigualdad y exclusión educativa en contexto de pandemia: Desafíos desde México}

La referencia a esta dramática historia en el presente artículo, tiene la intención de visibilizar los hilos, en algunos casos muy finos, y en otros no tanto, del proyecto colonial que continúa muy arraigado en nuestra región y que se evidencia con mayor fuerza a partir de una emergencia sanitaria.

En ese sentido, es necesario caracterizar el contexto neoliberal que por más de tres décadas moldeó al Sistema Educativo Nacional, desmantelando la noción de lo público, así como presentar las alternativas que el gobierno mexicano plantea para enfrentar la crisis y continuar con la recuperación de la soberanía educativa. Finalmente, en el presente artículo, se busca desentrañar los elementos que aún persisten en la burocracia educativa y que replica estrategias de privatización, aprovechando la situación de la pandemia.

\section{Neoliberalismo: el gran culpable}

Recurrimos a la memoria histórica para comprender y afrontar el nuevo contexto en el que nos encontramos, es decir, en medio de una crisis brutal, producto de un modelo económico que insiste en aniquilar cualquier expresión y organización comunitaria donde no vea reflejado el lucro.

La embestida neoliberal, al igual que la invasión europea a finales del siglo XV, viene acompañada de pestes y enfermedades ligadas a la propia dinámica capitalista.

La pandemia del COVID-19, con sus efectos devastadores, evidencia, como en ningún otro momento, la irracional forma de concebir todo como una mercancía, colocando a la mayoría de la población en un estado de vulnerabilidad jamás antes visto.

La pérdida de lo público en sus diferentes ámbitos tiene hoy graves repercusiones, y es particularmente en el espacio educativo donde se perciben las consecuencias más abrumadoras del desprecio a la educación en las últimas décadas.

Para ello, es necesario contextualizar cómo el modelo neoliberal se instauró en nuestro país incrementando aceleradamente las desigualdades y exclusiones educativas, las cuales son hoy el principal problema para enfrentar una crisis sanitaria como la que estamos viviendo.

La llegada del neoliberalismo es sin duda, el refrendo del colonialismo insertado en el pensamiento educativo. La exigencia mercantilista de un supuesto y único derecho, el privado, se expresó como la panacea en los discursos que adornaron los panfletos neoliberales de las últimas décadas.

Rev. Caminhos da Educação: diálogos, culturas e diversidades, Teresina, v. 2, n. 2, p.5-21, mai./ago. 2020 
A partir de la década de los ochenta y con mayor intensidad en los noventa, toda la riqueza pedagógica del país fue desplazada por directrices de organismos internacionales que consideraron pertinente sembrar la idea empresarial en el seno de las decisiones y concepciones de la educación. La Secretaría de Educación Pública (SEP) se llenó de "expertos" que sabían operar mejor los requerimientos de entidades como el Banco Mundial o el Fondo Monetario Internacional. En una carrera sin precedentes, cada sexenio se esforzó por implementar las reformas que estuvieran acordes a las recetas neoliberales.

En cada uno de los niveles educativos, las reformas tuvieron efectos diversos. Por un lado; se exigió universalizar la educación básica, piedra angular para el desarrollo de los países y de los individuos, ya que aseguró una supuesta mano de obra para el nuevo mercado laboral que está en boga y por el otro; promovió la privatización de la universidad.

Para poder llevar a cabo la redefinición de la educación básica y el desmantelamiento de la universidad pública el Banco Mundial impulsó la descentralización con los siguientes propósitos: Reorganización del gasto social en educación, flexibilización de los planes y programas de estudio y la intervención de nuevos actores en la toma de decisiones sobre la educación, especialmente la iniciativa privada.

La política de descentralización transfirió los "servicios educativos" a los estados, así como la reformulación de los contenidos y materiales y convirtió al magisterio en el principal blanco de todos los males que aquejan al Sistema Educativo Nacional, como ocurrió en la mal llamada Reforma Educativa de 2012, la cual fue acompañada de despidos masivos, represión y persecución a cientos de maestros y maestras de educación básica.

Por lo tanto, el proceso de descentralización benefició en gran medida a la iniciativa privada, ya que permitió la creación de condiciones en las que la educación formó parte de la lista de servicios, además de que eliminó restricciones legales para la participación de empresas o consorcios, permitiendo la supuesta libre competencia.

Lo anterior, eliminó la responsabilidad pública del Estado para convertirlo en "institución compensatoria" de estipendios, subsidios o vales a individuos, familias, instituciones u organizaciones que demuestren estar ejecutando las orientaciones de la descentralización y privatización de la educación. Esto permitió la libre actuación del mercado en el terreno educativo, ya sea entre las comunidades, entre los individuos o instituciones.

La descentralización, pues, fue la carta de presentación de la privatización de vastos sectores del sistema educativo, y el establecimiento de la

Rev. Caminhos da Educação: diálogos, culturas e diversidades, Teresina, v. 2, n. 2, p.5-21, mai./ago. 2020 


\section{Desigualdad y exclusión educativa en contexto de pandemia: Desafíos desde México}

participación obligatoria de los padres y madres de familia en los gastos educativos de sus hijos. Lo que se publicitó como autonomía de gestión, o incluso, democratización de la enseñanza, no fue sino la cara amable de un violento proceso de destrucción y desacreditación de la responsabilidad del Estado en la educación. Esta fue sustituida por el subsidio estatal a empresas educativas privadas, la apertura a la presencia empresarial en todos los niveles educativos, el cobro de cuotas y otras exigencias y condicionamientos en el conjunto del sistema educativo público. (Sosa, 2010: 45)

El sector de la educación superior fue el primero en modificar todo su aparato burocrático y académico, se impusieron cuotas en gran parte de las universidades públicas e impulsó el complejo empresarial creando pequeños organismos dentro de la universidad, los cuales, amparados en su legislación, legitimaron decisiones, de esa manera temas que tienen que ver con el presupuesto y su destino son tratados por un reducido grupo, éste a su vez, cumplió al pie de la letra las recomendaciones internacionales y reorganizó los programas de asignación de recursos financieros en estímulos económicos extraordinarios, generando la individualidad en la labor docente.

En ese sentido, el derecho a recibir una formación universitaria se restringió, creando un sistema elitista que permitió sólo a unos cuantos obtenerla; por ejemplo, más de trecientos mil aspirantes, en los últimos años se quedaron fuera de toda oportunidad de continuar estudios universitarios en universidades públicas y alrededor de 6.7 millones de jóvenes entre los 18 a 22 años no asisten a un servicio educativo. (SEP, 2019)

En general, los intentos por desmantelar el espacio público estuvieron acompañados por el constante uso de la violencia para minar cualquier intento de reorganización o reconstrucción del bien común.

El gobierno pasado de Enrique Peña Nieto (2012-2018) representó muy bien la etapa de podredumbre a la que fueron sometidas las instituciones estatales, no sólo por la corrupción, característica de los gobiernos neoliberales, sino por la represión y desaparición de miles de personas, entre las que destacan las y los jóvenes.

\section{Hacia una nueva transformación}

El cúmulo de todas estas injusticias y la persistente resistencia de diferentes sectores de la población llevaron a crear un amplio movimiento de izquierda encabezado por Andrés Rev. Caminhos da Educação: diálogos, culturas e diversidades, Teresina, v. 2, n. 2, p.5-21, mai./ago. 2020 
Manuel López Obrador quien logró un triunfo histórico en las elecciones electorales del 1 de julio de 2018 con más de 30 millones de votos.

El triunfo del presidente Andrés Manuel López Obrador representó el camino y trabajo de distintas generaciones que defendieron los derechos que alimentan todo sentido de lo público. Este legado histórico es agrupado en tres grandes transformaciones, la primera de ellas es la Guerra de Independencia (1810-1821), la segunda es la Guerra de Reforma (1858-1861), y la tercera es la Revolución Mexicana (1910-1917).

La conformación del Estado mexicano requirió de estas transformaciones populares que contribuyeron a la soberanía del país desafiando los proyectos coloniales e imperialistas de diferentes potencias internacionales. En nuestro contexto, el triunfo del 2018 significó una importante derrota para una clase privilegiada que se enriqueció en el periodo neoliberal.

El cambio de régimen, se concibe por lo tanto, como la cuarta transformación del país y su propósito es:

(...) construir una propuesta posneoliberal y convertirla en un modelo viable de desarrollo económico, ordenamiento político y convivencia entre los sectores sociales. Debemos demostrar que sin autoritarismo es posible imprimir un rumbo nacional; que la modernidad puede ser forjada desde abajo y sin excluir a nadie y que el desarrollo no tiene porqué ser contrario a la justicia social. (Plan Nacional de Desarrollo 2019-2024)

El triunfo de la izquierda en México trajo consigo una serie de planteamientos enfocados fundamentalmente a modificar el modelo económico del país y recuperar el Estado como garante de bienestar:

Durante décadas, la élite neoliberal se empeñó en reducir el Estado a un aparato administrativo al servicio de las grandes corporaciones y un instrumento coercitivo en contra de las mayorías. Su idea de que las instituciones públicas debían renunciar a su papel como rectoras e impulsoras del desarrollo, la justicia y el bienestar, y que bastaba "la mano invisible del mercado" para corregir distorsiones, desequilibrios, injusticias y aberraciones, fue una costosa insensatez. El Estado recuperará su fortaleza como garante de la soberanía, la estabilidad y el estado de derecho, como árbitro de los conflictos, como generador de políticas públicas coherentes y como articulador de los propósitos nacionales. (Plan Nacional de Desarrollo 2019-2024)

La recuperación del Estado se hace a través de una nueva concepción de política pública orientada a incrementar el gasto social, sobre la base, por un lado, de reducir los

Rev. Caminhos da Educação: diálogos, culturas e diversidades, Teresina, v. 2, n. 2, p.5-21, mai./ago. 2020 


\section{Desigualdad y exclusión educativa en contexto de pandemia: Desafíos desde México}

millonarios gastos de una burocracia parasitaria, siguiendo una política de austeridad republicana, inspirada en la postura del gobierno del presidente decimonónico Benito Juárez García, y por otro lado, desmantelando el sistema de corrupción que prevaleció sin mayor dificultad en los sexenios anteriores.

La recuperación de recursos a partir de estas dos políticas, permitieron lanzar programas sociales de bienestar que buscan reconstruir el tejido social y económico de la población más vulnerable.

Los programas contienen inversiones de megaproyectos en regiones muy poco atendidas en décadas pasadas; la entrega directa de recursos para reactivar la economía, especialmente la social y comunitaria; el rescate al campo; soberanía energética; educación en todos los niveles; salud; internet y construcción y rehabilitación de infraestructura, calles y caminos en las comunidades.

En ese sentido, los programas prioritarios que el gobierno federal implementó están dirigidos a la reactivación económica en zonas tradicionalmente abandonadas y se convierten en el mecanismo para crear las bases de un nuevo horizonte de país.

En el caso específico de la educación, los programas de bienestar se proyectaron en sentido totalmente opuesto a lo que venía funcionando, se invierte en la construcción de nuevas universidades, rehabilitación de los espacios escolares, becas universales, comedores estudiantiles, empleos con vínculo comunitario, entre otros.

\section{Educación y pandemia: alternativas}

En medio de estos procesos de transformación, el 28 de febrero de este año, se confirma el primer caso de coronavirus en México y para el 16 de marzo la Secretaría de Educación Pública (SEP), máxima autoridad educativa, anuncia la suspensión de clases a partir del 23 de marzo, bajo el entendido de que continuarán las actividades a distancia; comienza así el periodo de confinamiento que, al momento de redactar el presente artículo, no tiene fecha de conclusión.

Las medidas y el despliegue sanitario se enmarcan en los protocolos internacionales que se tienen para enfrentar la pandemia, con la característica fundamental de apego irrestricto a los derechos humanos, al menos desde la federación. No se implementó toques Rev. Caminhos da Educação: diálogos, culturas e diversidades, Teresina, v. 2, n. 2, p.5-21, mai./ago. 2020 
de queda o restricciones judiciales de movilidad, todo el trabajo de cuarentena fue voluntario, excepto las disposiciones legales respecto a establecimientos comerciales que por obvias razones debieron cerrar.

La comunicación constante del gobierno federal respecto al flujo de información del COVID-19 ayudó mucho a generar un tipo de conciencia en la mayoría de la población.

La oposición, por su parte, utilizó la situación para obtener capital político. Sus aspiraciones en los pocos estados que gobierna, es la de instaurar medidas sanitarias sobre la base del autoritarismo y la represión, sin embargo, sus ensayos e intentos de golpear al gobierno federal han fracasado.

No obstante, los efectos devastadores de la pandemia están muy relacionados con las enfermedades vinculadas a la mala alimentación, producto de la destrucción de la soberanía alimentaria, a las malas condiciones higiénico-sanitarias y los procesos de exclusión.

Por ejemplo, un estudio preliminar realizado por la Universidad Nacional Autónoma de México (UNAM) muestra que el 71 por ciento de los muertos por COVID-19, tenían apenas escolaridad primaria o inferior. Mientras que las ocupaciones de las víctimas se concentran en las siguientes categorías:

Servicios personales en establecimientos, Vendedores y trabajadores ambulantes, Servicios protección y vigilancia, Artesanos y trabajadores de fábricas, reparación y mantenimiento, Ayudantes, peones y similares No ocupados Profesionales, Ocupaciones no especificadas, Conductores, Empleados del sector público, No ocupado, jubilado o pensionado, No remunerado, amas de casa. (Hernández 2020: 6)

Si bien la peste comenzó entre un sector de la población privilegiada, en estos momentos se despliega entre los más pobres y con procesos de exclusiones más agudos.

Por lo anterior, el 16 de mayo el presidente presentó el ensayo titulado La nueva política económica en los tiempos del coronavirus en el que retoma los principales lineamientos del Plan Nacional de Desarrollo que sirven para apuntalar la crisis, para ello plantea la necesidad de pensar el crecimiento económico a partir de los siguientes elementos: democracia, justicia, honestidad, austeridad y bienestar, es decir, acordes al proyecto de nación.

Los cinco elementos de la nueva política económica son la base para impulsar los programas prioritarios, los cuales son esenciales para contribuir en la reconstrucción del Estado y crear las bases para transformar la situación de pobreza y exclusión que viven millones de mexicanos y mexicanas.

Rev. Caminhos da Educação: diálogos, culturas e diversidades, Teresina, v. 2, n. 2, p.5-21, mai./ago. 2020 


\section{Desigualdad y exclusión educativa en contexto de pandemia: Desafíos desde México}

El texto cobra relevancia porque deja claro que las prioridades del Estado son los más pobres y no el sector empresarial que comenzó a solicitar la condonación de impuestos, rescates o subsidios. (López Obrador, 2020).

Los programas reconfiguran la política pública, que hasta hace muy poco tiempo fue presa de una minoría. Se implementa, por lo tanto, una nueva redistribución de los recursos económicos, a partir de mantener la austeridad en las instituciones y eliminando intermediarios y a una abultada burocracia que se quedaba con una gran parte del presupuesto.

La puesta en práctica de una nueva administración del recurso público, se vuelve el motor, por un lado, para impulsar una democracia participativa en la que coloca a las comunidades en actores fundamentales de su propia transformación, y por el otro, dota al Estado de un propósito central: garantizar el bienestar del pueblo y para ello, deja de ser el "gestor de oportunidades" y se convierte en el garante de los derechos. (López Obrador, 2020).

Los programas prioritarios con la crisis y en contexto de pandemia son rediseñados concentrando la mayor atención en aquellos que más contribuyen a sostener a las familias: Pensión para el bienestar de los adultos mayores; bienestar de personas con discapacidad; Sembrando Vida; Programa de apoyo para el bienestar de los hijos de madres trabajadores; Becas Benito Juárez; Construcción de 100 universidades; la Escuela es Nuestra; Jóvenes Construyendo el Futuro; Tandas para el bienestar; Banco del Bienestar; Atención Médica y Medicamentos Gratuitos.

La transformación del país y seguramente la reconstrucción de lo que deje la tormenta llamada pandemia, surtirá efecto en aquellos lugares donde los programas sociales logren tener mayor efecto, sobre todo, en la medida en que generen aprendizajes colectivos en la toma de decisión de la propia comunidad. En estos momentos tan complicados: "La intervención del Estado resulta indispensable para la supervivencia de muchos" (López Obrador, 2020).

En el caso específico de la educación, me parecen importantes destacar, tres programas fundamentales que contribuyen a la construcción de alternativas bajo una nueva lógica de trabajo y responsabilidad colectiva: La Escuela es nuestra, la construcción de universidades y las becas universales.

Rev. Caminhos da Educação: diálogos, culturas e diversidades, Teresina, v. 2, n. 2, p.5-21, mai./ago. 2020 
El recurso para estos programas se entrega directamente a los beneficiarios, que se vuelven una especie de administradores y contralores comunitarios, poniendo en práctica la toma de decisiones que beneficien a sus comunidades, evitando la corrupción que floreció en el pasado, generando mayor certeza y evitando trámites burocráticos. El impacto en las comunidades es aún más grande, porque se reactivan las economías locales, se fomenta la participación de madres y padres de familia y se fortalece la organización popular. (SEP-La escuela es nuestra 2020)

Por lo tanto, es en los programas prioritarios de educación, que encontramos alternativas reales para la supervivencia, sustentabilidad y transformación de las comunidades. La escuela y la socialización se vuelven indispensables, a pesar de esta larga cuarentena. (UBBJ 2020).

Los programas, particularmente los de educación, son las alternativas más significativas que el gobierno del presidente Andrés Manuel López Obrador está realizando, ya que son el eje principal para contribuir en la recuperación de la soberanía del país y en específico reconstruir el espacio público, sobre todo en un momento de crisis económica que azota a gran parte del mundo.

\section{Educación y Pandemia: desafíos y retos}

Algunas cifras para contextualizar lo que ocurre con el Sistema Educativo Nacional y cómo éste contribuye a disminuir la pandemia son: 40.7 millones de personas que estudian o trabajan en alguno de los niveles educativos entraron en confinamiento, es decir, el 32.7 por ciento de la población total del país. (SEP)

Por ello, nos parece pertinente identificar los elementos que persisten en mantener la influencia del espacio privado y que, en contexto de pandemia, podrían estar alimentando la exclusión educativa que dejó el modelo neoliberal del país.

En ese sentido, 37.7 millones de estudiantes y 2 millones de docentes tuvieron que asumir el trabajo educativo a distancia, algo que ocurrió prácticamente en todo el mundo. Las herramientas utilizadas, son fundamentalmente las plataformas virtuales, pero también el uso de la televisión pública y los libros de texto, estos últimos para el nivel de educación básica.

Ahora bien, esta modalidad de trabajo virtual fue la respuesta que la pandemia impuso al mundo. Sin embargo, visibilizó y agudizó las diferentes exclusiones que viven millones de Rev. Caminhos da Educação: diálogos, culturas e diversidades, Teresina, v. 2, n. 2, p.5-21, mai./ago. 2020 


\section{Desigualdad y exclusión educativa en contexto de pandemia: Desafíos desde México}

estudiantes en países como México, producto del desmantelamiento que se generó en los últimos cuarenta años.

No obstante, el ingrediente clave en las actividades en casa es el uso del internet, una encuesta publicada por el Instituto Nacional de Estadística y Geografía, antes del confinamiento, menciona que existen en México 80. 6 millones de usuarios de internet, los cuales representan el 70.1\% de la población de seis años o más. (INEGI).

No obstante, cuando las cifras se leen por vivienda, reflejan un panorama más complejo, ya que se estima que sólo el 56.4\% de hogares en el país tienen conexión fija o móvil. La encuesta menciona que el principal medio para conectarse a Internet fue el celular, $95.3 \%$ de los usuarios lo utilizaron, mientras que el 33.2\% usó computadora portátil y $28.9 \%$ una computadora de escritorio. Finalmente, tres principales actividades con el uso de internet fueron el entretenimiento, la obtención de información y la comunicación.

Los datos muestran, que existe una desigualdad muy abismal entre los hogares que tienen conexión, los medios que se utilizan para navegar y el uso que se le da al Internet, por lo cual, es evidente que no existen las condiciones para trabajar de manera virtual en plataformas educativas, sobre todo a nivel básico de educación.

En tanto, las condiciones físicas en las que millones de estudiantes están intentando cumplir con sus actividades académicas, no son las más optimas.

La ONU-Habitad estima que 38.4 por ciento de la población en México habita en una casa en condiciones de hacinamiento o que carece de servicios mejorados como agua y saneamiento. (La Jornada, 14 de junio 2019).

La situación se vuelve más compleja cuando algún miembro de la familia es contagiado por COVID-19 y el asilamiento se tiene que cumplir en pleno hacinamiento, poniendo en riesgo a la familia, el barrio o la comunidad.

$\mathrm{Si}$ a lo anterior le sumamos el incremento de violencia que se genera en las familias, particularmente la de género, en tiempos de confinamiento; más la pobreza y en muchos casos el sostenimiento de la familia, a partir de un empleo informal, claramente afectado por la pandemia, podremos darnos cuenta de que la utópica idea de trabajo a distancia se desquebraja.

En cuanto a los y las docentes, sin previa formación, se han visto sometidos a utilizar plataformas, organizar programas y ejecutar tareas que rompen con su dinámica de enseñanza o en el peor de los casos han perdido su empleo, como está ocurriendo en Rev. Caminhos da Educação: diálogos, culturas e diversidades, Teresina, v. 2, n. 2, p.5-21, mai./ago. 2020 
instituciones privadas, que encuentran más factible trabajar de manera virtual con menos personal docente.

Por lo anterior, el espacio para enfrentar la pandemia y poder continuar con las actividades educativas, se convierte en el epicentro de las exclusiones y desigualdades y sin lugar a duda, no cumple con los mínimos requisitos para convertirse en el ideal del proceso de enseñanza-aprendizaje.

La pandemia, sin duda, no dio tregua parta crear muchas opciones en un sistema educativo en el que prevalecen históricas desigualdades, habría que mirar con más detalle las experiencias a nivel internacional, sin embargo, al menos en América Latina, la mayoría de los ministerios de educación se movieron por sendas muy similares y a más de cuatro meses de confinamiento, tendrían que replantearse el rumbo que tomaron.

El camino para desplegar el modelo educativo no puede pasar por alto las dificultades que enfrentan estudiantes, sus familias y docentes para poder cumplir con metas establecidas, que, dicho sea de paso, fueron proyectadas antes del confinamiento, menos en un contexto en el que proyecto de nación impulsado por un gobierno de izquierda busca recuperar la soberanía educativa vulnerada por esa basta operación ideológica impuesta en décadas pasadas.

Las recientes declaraciones del Secretario de Educación Pública, Esteban Moctezuma, en el marco de la XLIX Reunión Ordinaria del Consejo Nacional de Autoridades Educativas (CONAEDU) generaron preocupación entre diferentes actores políticos inmersos en la educación, ya que planteó que se mantendrá en el futuro, un modelo "híbrido" que combine lo presencial con el modelo a distancia. (SEP 2020)

Las implicaciones político-pedagógicas que ha dejado el largo ensayo de la cuarentena, sumado a las condiciones socioeconómicas descritas anteriormente, provocaron la reacción de los gremios magisteriales al denunciar que un modelo así, profundiza la discriminación, debido a la desigualdad de recursos materiales entre los estudiantes, es excluyente para personas con diferentes discapacidades, impacta en las relaciones laborales, ya que las plataformas promueven un tipo de control y de fiscalización de cómo y qué se trabaja, y, finalmente, mantiene la ruta de la privatización debido al jugoso negocio que tienen las grandes corporaciones como Google o Microsoft.

La asistencia que Microsoft y Google han brindado en el desarrollo del programa Aprende en Casa y el diseño del modelo híbrido que viene, no fue ni fortuita ni coyuntural. Es parte de un proceso de expansión

Rev. Caminhos da Educação: diálogos, culturas e diversidades, Teresina, v. 2, n. 2, p.5-21, mai./ago. 2020 


\section{Desigualdad y exclusión educativa en contexto de pandemia: Desafíos desde México}

corporativa que ha hallado en el sistema educativo nacional... Representa, además, la creación de un espacio de consumo dominante que favorece a Google, dado el apoyo recibido por parte de la SEP para incluir en su base de datos prácticamente a todos los trabajadores de la educación, a los cuales se les ha negado la posibilidad de elegir si quieren o no integrarse a dichas plataformas. Si bien la oferta de los servicios de Google ha sido en primera instancia gratuita, la lógica comercial con la cual se ha manejado en sistemas educativos del mundo ha causado fuertes críticas... En el contexto australiano, el uso generalizado de G Suite ha sido descrito por Anna Krien como un entrenamiento de consumidores de Google desde la infancia y un programa de fidelización de marca presentado como revolución educativa. (Jarquín 2020)

La información que obtienen estas corporaciones les permite crear un amplio mercado y una fuerte tendencia en espacios y sectores de la población, por lo tanto, son las ganadoras de la pandemia. Sin embargo, no es sólo la extensión mercantilista o la neo privatización de la educación, lo que llama la atención, es el replanteamiento pedagógico orientado bajo una nueva idea de competencia, sólo que ahora aparentemente invisible y virtual.

Lo cual trae como consecuencia el retorno de un modelo instrumental que racionaliza a partir de los parámetros impuestos por la corporación y coloca al docente en el estrecho papel de administrador.

El uso de la plataforma Clasroom de Google, utilizada ampliamente por docentes en México, retoma la esencia de todo aquello que fue duramente criticado por la pedagogía crítica del siglo pasado. Miguel Andrés Brenner, denuncia las implicaciones de trabajar con una plataforma como la de Google:

1. El docente no puede crear su propio diseño de clase, que viene ya pautado.

2. No hay posibilidad de retroalimentación entre alumnos-alumnos, docentes-docentes, alumnos-docentes

3. Desde el punto de vista pedagógico, para el docente, es un trabajo meramente individual, que no favorece la comunicación entre docentes, no permite la socialización o mirada de los contenidos por parte de otros docentes.

4. Permite trabajar por módulos o unidades a través de temas, pero no a través de problemáticas, con la complejidad de un trabajo crítico-creativocolaborativo desde una perspectiva ético-política.

Rev. Caminhos da Educação: diálogos, culturas e diversidades, Teresina, v. 2, n. 2, p.5-21, mai./ago. 2020 
5. Se pierde el trabajo personalizado en la relación alumno-docente y en la relación alumno-alumno.

6. Cada tarea es para hacer en casa, enviarla digitalmente al docente, quien luego hace una devolución, pero no se da virtualmente la dinámica dialógica como dentro de un espacio áulico.

7. Importa una constante revisión de los criterios pedagógico/didácticos referidos a la enunciación de explicaciones, textos y consignas.

8. Por otro lado, en la medida en que se apliquen "pruebas" o "tests" a los alumnos por opciones múltiples, ello apunta a una especie de tecnicismo-positivista propio de un enciclopedismo o modalidad bancaria tan criticada por Paulo Freire.

9. Otros problemas tienen que ver con la conectividad, con el tipo de instrumentos tecnológicos que cada alumno tiene, con el tener o no en el hogar un espacio propio para poder estudiar, con el acompañamiento o no de los padres y la calidad efectiva de dicho acompañamiento. (Brenner, 2020)

La inmensa operación económica e ideológica a la que fue sometida la educación en las últimas décadas intenta cobrar fuerza en una modalidad que antes de la pandemia era medianamente implementada, pero que ahora cobró gran auge: la extensión de la corporación en la escuela, convertida ésta en un espacio de promoción del lucro.

Por lo tanto, es erróneo llamar educación a distancia a un modelo de privatización, que como bien plantea el pedagogo brasileño Luis Carlos de Freitas, se trata de un oportunismo que se aprovecha de una crisis para terminar de introducir el mercado en la educación pública, por ello, no se trata de enseñanza a distancia, sino la introducción de una propaganda que en estos momentos lanzó a la improvisación a millones de madres, padres y docentes. (De Freitas 2020)

\section{Consideraciones finales: rostro y corazón}

En la filosofía de las culturas prehispánicas, particularmente la Náhuatl, los elementos esenciales para poder generar los procesos de socialización y la trasmisión de saberes fueron el rostro y el corazón.

El hombre maduro:

corazón firme como la piedra, corazón resistente como el tronco de un árbol;

rostro sabio, dueño de un rostro y un corazón, hábil y comprensivo. (León Portilla 1957)

Rev. Caminhos da Educação: diálogos, culturas e diversidades, Teresina, v. 2, n. 2, p.5-21, mai./ago. 2020 


\section{Desigualdad y exclusión educativa en contexto de pandemia: Desafíos desde México}

El rostro y el corazón simbolizan la conjunción de aquel que decide tomar la palabra para expresarse, de ahí que estos dos elementos sean los pilares del pensamiento educativo náhuatl, ambos inseparables. No puede haber hombre o mujer que no fragüe su sabiduría en la apropiación de un rostro y un corazón.

La invasión de América y la posterior colonización intentaron borrar ese rostro y corazón de los pueblos prehispánicos y afrodescendientes. Los saberes y conocimientos comunitarios fueron perseguidos porque atentaban contra el ideal de lo privado.

En pleno siglo XXI, el neoliberalismo actúa de la misma forma, persigue sabidurías populares que van en sentido contrario del principio de lucro, deja en perpetua vulneración a millones de personas, las deshumaniza y las coloca al filo de un abismo. El cocoliztli ${ }^{3}$ que padecemos hoy, sin duda es la consecuencia más grave de lo anterior.

Ahora, como en otras épocas, la educación debe ser el eje fundamental para mantener los principios de trabajo colectivo, de tejer entre las comunidades la solidaridad, el respeto y la defensa de lo que hacen y piensan. Es el pueblo, como bien lo menciona el presidente Andrés Manuel López Obrador, quien salva al pueblo.

Por ello, creemos que la apuesta fundamental para hacer realidad esa consigna es a través de la aportación de las escuelas, los docentes y la comunidad. La interacción de estos actores en el espacio escolar constituye los procesos de igualdad, a través de un tipo de formación que ponga como elemento central la justicia.

En ningún otro momento, como ahora, se hace evidente la reivindicación de la escuela.

Las consecuencias de la pandemia nos dejan una enseñanza: La escuela es el espacio y expresión del sentido comunitario con diversidades y distinciones, por lo tanto, debemos apostar a ella. No se trata de repetir los graves errores que deshumanizaron su sentir, sino para que siga siendo el espacio de socialización más importante que tienen las personas a lo largo de su vida.

En medio de la pandemia, aún prevalecen ideales corporativos entre la burocracia, sin embargo, México está pasando por un momento histórico muy importante en el que se plantean alternativas desde el Estado para proyectar a la educación como parte fundamental

\footnotetext{
${ }^{3}$ En náhuatl significa epidemia y era la manera de nombrar a la peste. Rev. Caminhos da Educação: diálogos, culturas e diversidades, Teresina, v. 2, n. 2, p.5-21, mai./ago. 2020
} 
de la soberanía del país. Sin duda, estas alternativas van en sintonía de la filosofía náhuatl: dotar a la educación con rostro y el corazón

\section{Referencias}

Brenner Miguel Andrés, (2020) "Google classroom, la cuarentena y el silencio como ausencia pedagógica”. Educación Futura. http://www.educacionfutura.org/google-classroom-lacuarentena-y-el-silencio-como-ausencia-pedagogica/ (Consultado el 10 de mayo de 2020)

De Freitas Luis Carlos. "Educação e Pandemia: a EAD e os riscos do modelo empresarial" Mariana Conti. 14/04, terça feira, às 19 hs. https://www.youtube.com/watch?v=XW_856dR8RI (Consultado el 10 de mayo de 2020)

De Sousa Santos, Boaventura (2010) Descolonizar el saber, reinventar el poder. Montevideo, TRILCE.

Gobierno de México. Plan Nacional de Desarrollo. Diario Oficial de la Federación. https:/ /www.dof.gob.mx/nota_detalle.php?codigo $=5565599 \&$ fecha $=12 / 07 / 2019$

(Consultado el 20 de junio de 2020)

Gómez Mena, Carolina (2019) "Casi 40\% de la población de México vive en condiciones de hacinamiento: ONU-Habitat". La Jornada. https://www.jornada.com.mx/2019/06/14/sociedad/034n2soc (Consultado el 20 de junio de 2020)

Hernández, Héctor. (17 de junio de 2020). Mortalidad por covid-19 envMéxico. Notas preliminares para un perfil sociodemográfico. Notas de coyuntura del crim No. 36, México, crim-unam, $7 \mathrm{pp}$.

INAH. Código Mendoza. Instituto Nacional de Antropología e Historia. https://www.codicemendoza.inah.gob.mx/index.php?lang=spanish (Consultado el 20 de junio 2020)

INEGI. Comunicado de prensa número 103/20. Encuesta nacional sobre disponibilidad y uso de tecnologías de la información en los hogares, 2019. Instituto Nacional de Estadística y

Geografía.

https://www.inegi.org.mx/contenidos/saladeprensa/boletines/2020/Otr'TemEcon/END UTIH_2019.pdf (Consultado el 20 de junio de 2020)

Jarquín Ramírez, Mauro (2020). "Implicaciones de un modelo híbrido en educación". La Jornada. https://www.jornada.com.mx/2020/07/26/opinion/018a2pol (Consultado el 20 de junio de 2020)

León Portilla, Miguel (Mayo de 1957) "Ixtlamachiliztli: dar sabiduría a los rostros ajenos" Vol. XI. Número 9. México, UNAM en https://www.revistadelauniversidad.mx/download/fb5347dc-781f-4623-8327$\underline{895682105 e 6 e ? f i l e n a m e=i x t l a m a c h i l i z t l i-d a r-s a b i d u r i a-a-l o s-r o s t r o s-a j e n o s}$

México. UNAM.

(1963) Visión de los vencidos: relaciones indígenas de la conquista.

Rev. Caminhos da Educação: diálogos, culturas e diversidades, Teresina, v. 2, n. 2, p.5-21, mai./ago. 2020 


\section{Desigualdad y exclusión educativa en contexto de pandemia: Desafíos desde México}

Lopez Obrador, Andrés. (2020) "La nueva política económica en tiempos de coronavirus" https://lopezobrador.org.mx/wp-content/uploads/2020/05/LA-NUEVAPOLI $\% C C \% 81 T I C A-E C O N O \% C C \% 81$ MICA-EN-LOS-TIEMPOS-DELCORONAVIRUS-15-MAYO-2020.pdf (Consultado el 10 de junio de 2020)

SEP. (2020) Respuestas de las Instituciones Públicas de Educación Superior en México para enfrentar la crisis del COVID-19. Secretaría de Educación Pública. http://www.anuies.mx/media/docs/avisos/pdf/200417115709VF_ACCIONES_SES_C OVID_19_ANUIES.pdf

SEP. Sistema Educativo de los Estados Unidos Mexicanos. Principales cifras 2018-2019. Secretaría de Educación Pública. https://www.planeacion.sep.gob.mx/Doc/estadistica_e_indicadores/principales_cifras/pr incipales_cifras_2018_2019.pdf (Consultado el 20 de junio de 2020)

Sosa, Raquel (2010) Hacia la recuperación de la soberanía educativa en América Latina: conciencia crítica y programa. México, UNAM.

Rev. Caminhos da Educação: diálogos, culturas e diversidades, Teresina, v. 2, n. 2, p.5-21, mai./ago. 2020 